\title{
Carbenoxolone has the potential to ameliorate acute incision pain in rats
}

\author{
SHUZHEN DONG, KAI ZHANG and YISA SHI \\ Department of Anesthesiology, The Second Hospital of Lanzhou University, Lanzhou, Gansu 730030, P.R. China
}

Received August 7, 2020; Accepted January 7, 2021

DOI: $10.3892 / \mathrm{mmr} .2021 .12159$

\begin{abstract}
Carbenoxolone (CBX) is primarily used to relieve various types of neuropathic and inflammatory pain. However, little is known concerning the role of CBX in acute pain and its functional mechanisms therein and this was investigated in the present study. Rats underwent toe incision and behavioral tests were performed to assess mechanical hypersensitivity. The expression levels of pannexin 1 (Px1) and connexin 43 (Cx43) were detected using western blot analysis $2,4,6$ or $24 \mathrm{~h}$ after toe incision, and the expression of TNF- $\alpha$, IL-1 $\beta$ and P substance (SP) was determined by ELISA; Px1 and Cx43 expression was also examined by immunofluorescence staining. At 2 , 6 and $12 \mathrm{~h}$ post-toe incision, the postoperative pain threshold was significantly reduced, which was subsequently recovered at 2 and $6 \mathrm{~h}$ post-surgery following pretreatment with CBX or pannexin 1 mimetic inhibitory peptide. CBX reduced Px1 levels at 4 and $24 \mathrm{~h}$ post-incision. However, $\mathrm{Cx} 43$ levels were reduced by CBX as little as $2 \mathrm{~h}$ post-surgery. Furthermore, CBX not only distinctly decreased the levels of Px1 and Cx43, but also reduced the co-localization of Px1 or Cx43 with glial fibrillary acidic protein, $2 \mathrm{~h}$ after incision. It was also observed that the protein levels of inflammatory makers (IL-1 $\beta$, SP and TNF- $\alpha$ ) showed a tendency to decline at 2, 4, 6 and $24 \mathrm{~h}$ after incision. Collectively, the expression of Px 1 and Cx43 in astrocytes may be involved in pain behaviors diminished by $\mathrm{CBX}$, and CBX potentially reduces acute pain by decreasing Px1 and Cx43 levels. Px1 and Cx43 from spinal astrocytes may serve important roles in the early stages and maintenance of acute pain, while preoperative injection of CBX has the potential to relieve hyperalgesia.
\end{abstract}

\section{Introduction}

Postoperative pain is characterized by acute pain that occurs immediately after surgery. The duration is usually no more

Correspondence to: Dr Yisa Shi, Department of Anesthesiology, The Second Hospital of Lanzhou University, 82 Cuiyingmen Road, Chengguan, Lanzhou, Gansu 730030, P.R. China

E-mail: shiyscat@163.com

Key words: pannexin-1, incision pain, acute pain, astrocytes than 7 days, but when poorly controlled, can develop into chronic pain syndrome, which not only causes physical and mental distress to the patient, but also increases economic burden on the family and society in general (1). In previous years, the role of glial cells in the transformation from acute to chronic pain, as well as the maintenance of slow pain, has received increasing attention $(2,3)$.

Gap junction (GJ)-mediated coupling occurs among satellite glial cells and is facilitated by GJ proteins composed of two hemichannels (HC), including connexins (Cx), pannexin (Px) and innexin (Inx) (4,5). Previous studies have indicated that Px1 affects the activation of astrocytes by regulating the release of ATP and the flow of calcium (6,7). At the neuronal membrane, ATP binds to the ATP receptor to induce the production of glutamic acid. ATP and glutamic acid have been identified as important molecules for the transmission of pain signals in the spinal dorsal horn. Px1 is expressed in a variety of tissues, and mediates the propagation of the calcium wave, which may influence the maintenance of pain (8). As such, Px1 plays a vital role in the transmission and maintenance of pain. Previous studies reported that Px1 activated inflammasome signaling, and that its inhibition reduced chronic pain and hypersensitivity in glial fibrillary acidic protein (GFAP)-positive glia cells (9-11), while its upregulation promoted the development of chronic pain (9).

Intrathecal administration of carbenoxolone (CBX), a gap junction blocker, can suppress central sensitization of existing neuropathic pain (12). CBX has been reported to suppress Cx expression, including that of $\mathrm{Cx} 43$ (13). Cxs are involved in inducing and maintaining chronic pain (14); Cx43 forms a hemichannel, resulting in the release of mediators, such as ATP and glutamate, into the extracellular space, which activate nociceptive neurons to induce pain. Moreover, non-neuronal cells also initiate pain through the release of cytokines triggered by extracellular ATP binding to its receptor (15). However, another study revealed that a reduction in the expression of one GJ protein could cause an increase in that of another (16). Therefore, the present study aimed to determine whether Px1 and Cx43 are involved in the formation of acute pain, and whether this can be relieved by the intrathecal injection of CBX.

\section{Materials and methods}

Animals. Sprague-Dawley rats of clean grade $(n=102$; weight, 200-250 g, male) were provided by the Laboratory 
Animal Center of Lanzhou Veterinary Research Institute, Chinese Academy of Agricultural Sciences. All experiments were performed in accordance with the guidance of The International Association for the Study of Pain (17), and approved by the Ethics Committee of The Second Hospital of Lanzhou University (approval no. D2019-064; Lanzhou, China). The rats were kept at $22-26^{\circ} \mathrm{C}$ with a relative humidity of $45-60 \%$ and a $12 \mathrm{~h}$ light/dark cycle. They had free access to food and water. The rats were randomly divided into the following five groups: i) Control group $(\mathrm{C}, \mathrm{n}=6)$; ii) incision pain model group (IP, $\mathrm{n}=24$ ); iii) normal saline group (NS, $10 \mu \mathrm{l}, \mathrm{n}=24)$; iv) $\mathrm{CBX}$ treatment group (CBX, $100 \mu \mathrm{M}, 10 \mu \mathrm{l}$, $\mathrm{n}=24)$; and v) pannexin-1 mimetic inhibitory peptide treatment group (10panx, $100 \mu \mathrm{M}, 10 \mu \mathrm{l}, \mathrm{n}=24)$. CBX and 10panx were purchased from APExBIO Technology LLC. With the exception of the normal group, the rats in each group were further divided into four groups: i) $2 \mathrm{~h}$ post-surgery (n=6); ii) $4 \mathrm{~h}$ post-surgery ( $\mathrm{n}=6)$; iii) $6 \mathrm{~h}$ post-surgery $(\mathrm{n}=6)$; and iv) $24 \mathrm{~h}$ post-surgery $(n=6)$. The experimental process is shown in Fig. 1. The rationale for choosing the time points was based on previous literature $(5,12,18)$. In the present study, rats were injected with $50 \mu \mathrm{M}(0.3 \mu \mathrm{g} / 10 \mu \mathrm{l})$ or $100 \mu \mathrm{M}(0.6 \mu \mathrm{g} / 10 \mu \mathrm{l})$ $\mathrm{CBX}$ intragastric, and the pain relief of acute IP was observed. Finally, it was verified that $100 \mu \mathrm{M}(0.6 \mu \mathrm{g} / 10 \mu \mathrm{l}) \mathrm{CBX}$ could significantly relieve acute IP, so this concentration of CBX was selected. The rats in the control group received no treatment. The baseline mechanical withdrawal threshold (MWT) was measured for each rat. After MWT and thermal withdrawal latency (TWL) were measured at different time points, the rats were sacrificed by cervical dislocation under anesthesia with $1 \%$ pentobarbital sodium (intraperitoneal) and the lumbar enlargement of the spinal cord was removed.

Animal model establishment. A rat model of acute IP was established using the Brennan method (19). The rats were anesthetized in a transparent anesthesia box with $2 \%$ sevoflurane inhalation in oxygen and then fixed on the operating table. A longitudinal incision of $\sim 1 \mathrm{~cm}$ was made $0.5 \mathrm{~cm}$ from the proximal heel of the foot to the toe. After cutting the skin and fascia, the muscle was located with forceps and cut longitudinally to avoid injury and comprising muscle integrity during the procedure. After hemostasis, the skin was sutured with two stitches of 3-0 fine thread, keeping an even distance between the two stitches.

\section{Behavioral examination}

$M W T$ detection. The rats were placed in a plexiglass box with a metal mesh bottom for $30 \mathrm{~min}$. Once the rats had acclimated to these conditions (remaining relatively quiet), a series of standardized von Frey filaments were inserted through the metal grid, and used to vertically stimulate the skin near the incision of the left posterior claw, so that the filaments were continuously bent and maintained for $6-8 \mathrm{sec}$. The time interval between each stimulation was $>30 \mathrm{sec}$, and the initial stimulation intensity was $2.0 \mathrm{~g}$. If three of the five stimuli instigated a rapid foot retraction or licking response, pain ' $\mathrm{X}$ ' was recorded and a lower level of stimulation intensity was administered. On the contrary, if $<$ three stimuli instigated this response, it was denoted as ' $\mathrm{O}$ ', and a stimulus intensity of a higher level was used. When a different reaction occurred, the MWT was measured another four times in sequence. The last stimulus was recorded after six measurements. If the stimulus intensity was 15 or $0.4 \mathrm{~g}$, the MWT value was directly denoted as 15 or $0.4 \mathrm{~g}$. The investigators were blinded to the experimental grouping of the animals.

TWL. TWL was determined in accordance with the Hargreaves method (13). The rats were first allowed to adapt to the organic glass box for 10-15 min. Thereafter, as a heat source, a thermal radiation stimulator was placed under the glass plate next to the incision of the rear claw. The shrinkage leg incubation period was determined as the time from the initial heat source stimulation to the retraction of the leg. The duration time of each stimulation was $30 \mathrm{sec}$, and the test was performed three times using the same stimulus intensity (measurement interval, $5 \mathrm{~min}$ ). The investigators were blinded to the experimental grouping of the animals.

Western blot analysis. After behavioral examination at 2 , 4,6 or $24 \mathrm{~h}$, the rats were sacrificed by cervical dislocation under anesthesia intraperitoneally with $1 \%$ pentobarbital sodium ( $50 \mathrm{mg} / \mathrm{kg}$ body weight), and the lumbar enlargement of the spinal cord was retrieved. The lumbar enlargement was lysed on ice with RIPA Lysis Buffer (cat. no. R0020, Solarbio) for $15 \mathrm{~min}$ and centrifuged at $4,025 \mathrm{x} \mathrm{g}$ at $4^{\circ} \mathrm{C}$ for $30 \mathrm{~min}$. Part of the supernatant was taken and the protein concentration was measured by BCA method; $4 \mathrm{X}$ loading buffer was added into the rest of the supernatant for western blotting. The supernatant was incubated in boiling water for $5 \mathrm{~min}$. The mass of protein loaded per lane was $40 \mu \mathrm{g}$. The proteins were then subjected to $10 \%$ sodium dodecyl sulfate polyacrylamide gel electrophoresis, and then transferred onto a PVDF membrane, which was subsequently blocked for $2 \mathrm{~h}$ at room temperature using 5\% skimmed milk powder dissolved into TBST with $0.05 \%$ Tween-20 (TBST) solution. The membrane was incubated at $4{ }^{\circ} \mathrm{C}$ overnight with the following antibodies: Anti-Px1 antibody (1:800; cat. no. ab139715; Abcam), anti-Cx43 antibody (1:1,000; cat. no. ab11369; Abcam) and anti-GADPH (1:5,000; cat. no. sc-365062; Santa Cruz Biotechnology, Inc.). Then, the membrane was rinsed three times with TBST for $10 \mathrm{~min}$ each, and incubated with HRP-conjugated goat anti-mouse IgG (cat. no. 15014) and goat anti-rabbit IgG (cat. no. 15015) secondary antibodies (1:8,000; ProteinTech Group, Inc.). The protein bands were visualized using ECL luminescent solution (Biosharp Life Sciences), and the gray value was analyzed using ImageJ software v1.52r (National Institutes of Health).

Immunofluorescence (IF) assay. An IF assay was performed $2 \mathrm{~h}$ post-surgery. After the behavioral test, the heart was exposed under deep anesthesia and the aorta was catheterized by apical puncture, followed by sequential infusion of $250 \mathrm{ml}$ normal saline and $500 \mathrm{ml}$ paraformaldehyde (4\%). The lumbago was exposed and removed, immersed in $4 \%$ paraformaldehyde overnight, and then sequentially immersed in $20 \%$ and $30 \%$ sucrose solution for frozen sectioning. After drying at room temperature, the sections (3-5 $\mu \mathrm{m}$ ) were fixed with $4 \%$ paraformaldehyde for $15 \mathrm{~min}$ at room temperature, and then washed with PBS three times for $5 \mathrm{~min}$ each. Then, the sections were permeabilized with $0.5 \%$ TritonX-100 for $10 \mathrm{~min}$ at room 


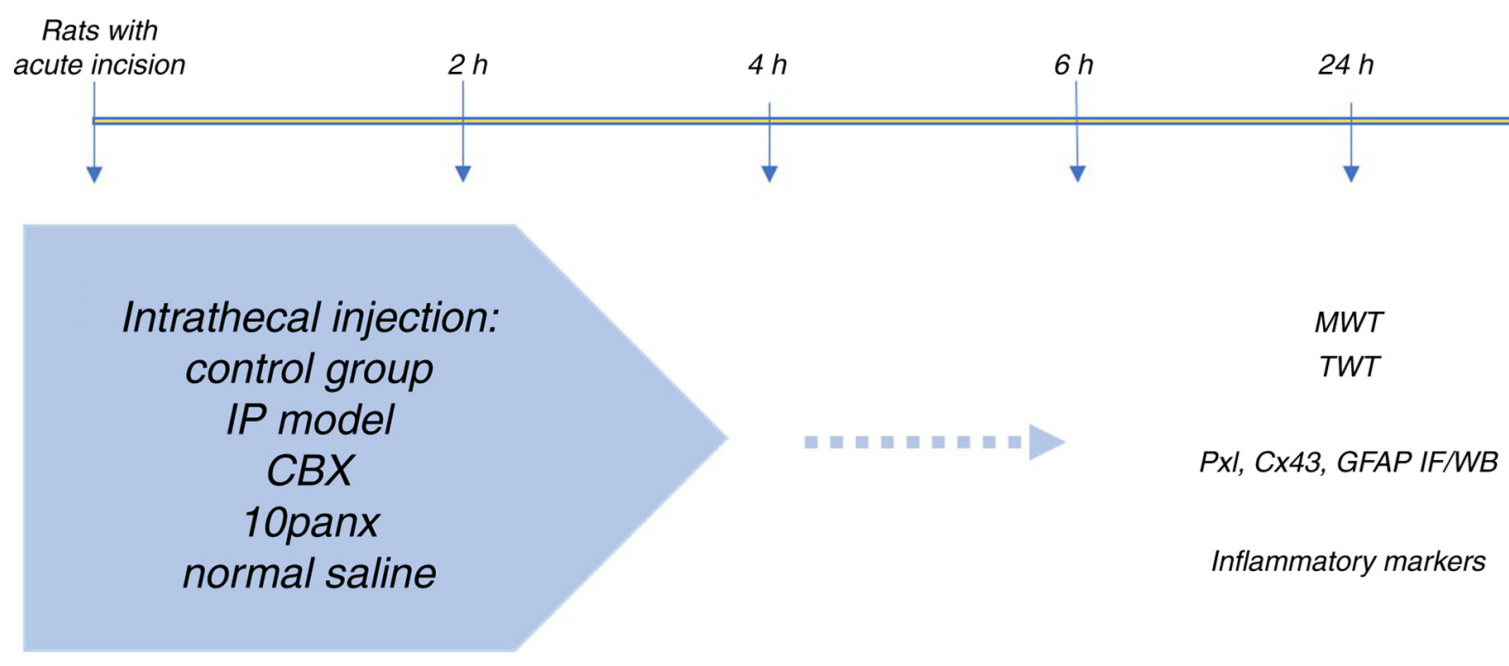

Figure 1. Flow chart of the experimental process. The rat model of acute incision was established and CBX, 10panx or normal saline administrated. IP, incision pain; CBX, carbenoxolone; 10panx, pannexin-1 mimetic inhibitory peptide; MWT, mechanical withdrawal threshold; TWL, thermal withdrawal latency; Px1, pannexin 1; Cx43, connexin 43; GFAP, glial fibrillary acidic protein; IF, immunofluorescence; WB, western blotting.

temperature and washed with PBS three times for 5 min each. The sections were then immersed in citrate antigen retrieval solution, heated for $15 \mathrm{~min}$ at $95-100^{\circ} \mathrm{C}$, washed with PBS and blocked with $10 \%$ bovine serum albumin for $1 \mathrm{~h}$ at room temperature.

Px1 and GFAP double staining, along with Cx43 and GFAP double staining (Px1, Cx43, GFAP, 1:100. GFAP, Affinity Biosciences), were performed. The sections were incubated at $4^{\circ} \mathrm{C}$ overnight with the primary antibodies (anti-GFAP, 1:100; cat. no. BF0345; Affinity Biosciences), followed by incubation for $1 \mathrm{~h}$ at $37^{\circ} \mathrm{C}$ with FITC-conjugated goat anti-mouse IgG (H+L) (cat. no. S0007; 1:100; Affinity Biosciences) and goat anti-rabbit IgG (H+L) secondary antibodies (cat. no. S0015; 1:100; Affinity Biosciences). The sections were stained with DAPI solution at room temperature for $8 \mathrm{~min}$ and sealed with immunofluorescence quencher at room temperature (Beijing Solarbio Science \& Technology Co., Ltd.) and images were captured under a fluorescence microscope (magnification, x200; Olympus Corporation).

ELISA. The levels of TNF- $\alpha$ (cat. no. D731168), $1 \mathrm{~L}-1 \beta$ (cat. no. D731007) and P substance (SP; cat. no. D751030) were analyzed using ELISA kits (all purchased from Sangon Biotech Co., Ltd.), according to the manufacturer's instructions. Blank, standard and sample wells were set. For each sample, $50 \mu \mathrm{l}$ standard substance was added to each standard well, and the sample was diluted five times with sample diluent prior to its addition to the sample well. After $30 \mathrm{~min}$ at $37^{\circ} \mathrm{C}$, each well was washed five times. Color developing agents $\mathrm{A}$ $(50 \mu \mathrm{l})$ and $\mathrm{B}(50 \mu \mathrm{l})$ were then sequentially added to each well. Finally, the reaction was terminated by adding $50 \mu \mathrm{l}$ termination reagent to each well. The absorption value of each well was measured using a microplate reader at a wavelength of $450 \mathrm{~nm}$ (Tecan Group, Ltd.).

Statistical analysis. Statistical analyses were performed using SPSS 20.0 software (IBM Corp), and the results are expressed as the mean \pm SD. Figures were created using GraphPad Prism 7.0 software (GraphPad Software, Inc.). The experimental data of MWT and TWL among different groups were analyzed using mixed ANOVA, followed by Bonferroni's post hoc test. Comparisons among multiple groups were performed using one-way ANOVA, followed by Tukey's post hoc test. If the data had marked deviations from normality, Friedman's test was performed, followed by Bonferroni-Dunn's test. $\mathrm{P}<0.05$ was considered to indicate a statistically significant difference.

\section{Results}

Effects of $C B X$ on $M W T$ and TWL in rats with IP. There were no significant differences between MWT and TWL among the different groups. The MWT was significantly decreased in the IP and NS groups compared with the control group $(\mathrm{P}<0.001$; Table I), although there was no significance change between the IP and NS groups at any time point. Compared with the IP group, the MWT and TWL were significantly increased by CBX or 10panx 2 and $4 \mathrm{~h}$ after surgery $(\mathrm{P}<0.001$; Tables I and II). In addition, TWL was increased by CBX and 10panx $6 \mathrm{~h}$ after surgery compared with the IP group $(\mathrm{P}<0.001$; Table II). The MWT in the 10panx group was similar to that in the CBX group. However, the TWL in the 10panx group was higher than that in the CBX group 2 and 24 h post-surgery $(\mathrm{P}<0.05$; Table II). Therefore, the results suggested that CBX significantly increased the pain threshold of rats experiencing acute IP, and that CBX enhanced the heat pain threshold to a higher degree than the MWT.

CBX significantly decreases $P x 1$ and CX43 expression. After surgery, Px1 was significantly upregulated in the spinal cords of the IP rats compared with the control group $(\mathrm{P}<0.001$; Fig. 2). Moreover, Px1 levels in the IP rats showed a greater increase at 6 and 24 h post-surgery than at 2 and $4 \mathrm{~h}$. Thus, to a certain extent, Px1 was significantly increased in a time-dependent manner. Compared with NS treatment, CBX significantly reduced $\mathrm{Px} 1$ levels at 4 and $24 \mathrm{~h}(\mathrm{P}<0.05)$. However, the decrease in Px1 was most significant at $4 \mathrm{~h}$ post-surgery $(\mathrm{P}<0.001)$. 10panx administration significantly reduced Px1 levels at 2, 4, 6 and 24 h compared with the NS 
Table I. MWT at different time points of each group.

\begin{tabular}{|c|c|c|c|c|c|}
\hline \multirow[b]{2}{*}{ Group } & \multirow[b]{2}{*}{ Baseline, $g$} & \multicolumn{4}{|c|}{ MWT } \\
\hline & & $2 \mathrm{~h}$ & $4 \mathrm{~h}$ & $6 \mathrm{~h}$ & $8 \mathrm{~h}$ \\
\hline $\mathrm{C}$ & $12.10 \pm 3.47$ & $12.78 \pm 3.44$ & $12.00 \pm 3.31$ & $12.11 \pm 2.22$ & $12.14 \pm 2.73$ \\
\hline IP & $12.10 \pm 3.47$ & $2.03 \pm 0.97^{\mathrm{a}}$ & $0.97 \pm 0.77^{\mathrm{a}}$ & $1.73 \pm 1.13^{\mathrm{a}}$ & $8.05 \pm 3.59$ \\
\hline NS & $12.78 \pm 3.44$ & $1.92 \pm 1.09^{\mathrm{a}}$ & $0.63 \pm 0.35^{\mathrm{a}}$ & $2.31 \pm 0.63^{\mathrm{a}}$ & $5.55 \pm 1.96$ \\
\hline CBX & $12.00 \pm 3.31$ & $6.02 \pm 1.06^{\mathrm{b}}$ & $7.95 \pm 1.48^{\mathrm{b}}$ & $3.39 \pm 1.72$ & $6.34 \pm 1.16$ \\
\hline 10panx & $10.39 \pm 3.59$ & $7.00 \pm 1.63^{b}$ & $5.94 \pm 1.73^{b}$ & $2.43 \pm 0.93$ & $7.95 \pm 1.58$ \\
\hline
\end{tabular}

Results are presented as the mean $\pm \mathrm{SD}(\mathrm{n}=6)$. ${ }^{\mathrm{a}} \mathrm{P}<0.001 \mathrm{vs}$. $\mathrm{C}$ group; ${ }^{\mathrm{b}} \mathrm{P}<0.001 \mathrm{vs}$. IP group. MWT, mechanical withdrawal threshold; $\mathrm{C}$, control group; IP, incision pain; NS, normal saline; CBX, carbenoxolone; 10panx, pannexin-1 mimetic inhibitory peptide.

Table II. TWL at different time points of each group.

\begin{tabular}{|c|c|c|c|c|c|}
\hline \multirow[b]{2}{*}{ Group } & \multirow[b]{2}{*}{ Baseline, $\mathrm{g}$} & \multicolumn{4}{|c|}{ TWL } \\
\hline & & $2 \mathrm{~h}$ & $4 \mathrm{~h}$ & $6 \mathrm{~h}$ & $8 \mathrm{~h}$ \\
\hline $\mathrm{C}$ & $25.13 \pm 0.69$ & $24.73 \pm 0.81$ & $25.01 \pm 0.98$ & $25.14 \pm 0.53$ & $24.64 \pm 0.68$ \\
\hline IP & $25.13 \pm 0.69$ & $5.54 \pm 1.15^{\mathrm{a}}$ & $3.32 \pm 0.36^{\mathrm{a}}$ & $5.98 \pm 0.55^{\mathrm{a}}$ & $9.17 \pm 1.99^{\mathrm{a}}$ \\
\hline NS & $24.73 \pm 0.81$ & $6.07 \pm 0.58^{a}$ & $3.32 \pm 0.57^{a}$ & $6.39 \pm 1.32^{\mathrm{a}}$ & $9.12 \pm 0.46^{a}$ \\
\hline CBX & $25.27 \pm 0.69$ & $13.76 \pm 3.33^{\mathrm{b}}$ & $17.08 \pm 2.73^{b}$ & $13.53 \pm 3.73^{b}$ & $18.74 \pm 0.90$ \\
\hline 10panx & $24.68 \pm 0.65$ & $18.57 \pm 2.64^{\mathrm{b}, \mathrm{c}}$ & $15.82 \pm 2.42^{\mathrm{b}}$ & $13.77 \pm 2.70^{\mathrm{b}}$ & $20.96 \pm 2.06^{c}$ \\
\hline
\end{tabular}

Results are presented as the mean $\pm \mathrm{SD}(\mathrm{n}=6) .{ }^{\mathrm{a}} \mathrm{P}<0.001$ vs. $\mathrm{C}$ group; ${ }^{\mathrm{b}} \mathrm{P}<0.001 \mathrm{vs}$. IP group; ${ }^{\mathrm{c}} \mathrm{P}<0.05$ vs. $\mathrm{CBX}$ group. TWL, thermal withdrawal latency; C, control group; IP, incision pain; NS, normal saline; CBX, carbenoxolone; 10panx, pannexin-1 mimetic inhibitory peptide.

group $(\mathrm{P}<0.05)$, which was most significant at 24 h post-surgery $(\mathrm{P}<0.05)$. This could indicate that $\mathrm{CBX}$ and 10panx reduce Px1 levels via different molecular mechanisms. Furthermore, toe incision postoperatively increased $\mathrm{Cx} 43$ expression levels at 2, 6 and 24 h compared with the control group (Fig. 2). CBX significantly reduced the levels of $\mathrm{Cx} 43$ at $2 \mathrm{~h}$ post-surgery, but led to an increase in these levels at 4, 6 and $24 \mathrm{~h}$ after surgery. 10panx significantly decreased Cx43 levels at 4, 6 and $24 \mathrm{~h}$ post-surgery compared with the NS group, suggesting that CBX pretreatment decreased Cx43 expression shortly after surgery.

Co-localization of PxI/CX43 and GFAP. An IF assay was performed to analyze the co-localization of Px1 or $\mathrm{Cx} 43$ with GFAP $2 \mathrm{~h}$ after incision. Green indicates Px1 expression in Fig. 3 and Cx43 expression in Fig. 4, red indicates astrocytes, while yellow indicates the co-localization of Px1 or Cx43 with GFAP. As shown in Fig. 3, the co-expression of Px1 and GFAP was most apparent in the IP group. Simultaneously, CBX markedly reduced the co-expression of Px1 and GFAP in the intumescentia lumbalis compared with the IP group, which was higher than that in the 10panx group. It was also found that CBX treatment led to a slight decrease in the co-localization of Cx43 and GFAP compared with the IP group (Fig. 4). Additionally, 10panx treatment appeared to lead to a more pronounced reduction in the co-localization of Cx43 and GFAP compared with CBX group. Therefore, the results suggested that $\mathrm{CBX}$ induced astrocytes to express low levels of Cx43 and Px1 in rats with toe incision.

$C B X$ reduces the expression levels of inflammatory markers at different time points after toe incision. Glia cells contribute to pain effects via the synthesis and release of a variety of inflammatory mediators, including IL-1 $\beta$ and TNF- $\alpha(20,21)$. In the present study it was observed that IL-1 $\beta$ expression levels were markedly increased at 2, 4, 6 and $24 \mathrm{~h}$ post-surgery in the IP group compared with the control group $(\mathrm{P}<0.05)$, while the protein levels of SP were increased 2, 6 and $24 \mathrm{~h}$ after surgery in the IP group $(\mathrm{P}<0.05)$. However, incision only induced a significant increase in TNF- $\alpha$ at the $2 \mathrm{~h}$ time point $(\mathrm{P}<0.05)$. These results implied that toe incision can induce an inflammatory response $2 \mathrm{~h}$ after surgery. Although a decline in the levels of inflammatory-related markers was observed following CBX pretreatment (Fig. 5), this effect was not significant. Notably, 10panx treatment led to a decrease in IL-1 $\beta$ levels at $6 \mathrm{~h}$ $(\mathrm{P}<0.01)$ and $24 \mathrm{~h}(\mathrm{P}<0.05)$ compared with the IP group, as well as a decrease in SP levels at 2 and $6 \mathrm{~h}(\mathrm{P}<0.05)$, demonstrating that the inhibition of Panx1 could effectively reduce the levels of pro-inflammatory factors increased by incision surgery.

\section{Discussion}

The preferred method of treatment for relieving postoperative pain currently involves the use of opioids, which are associated 

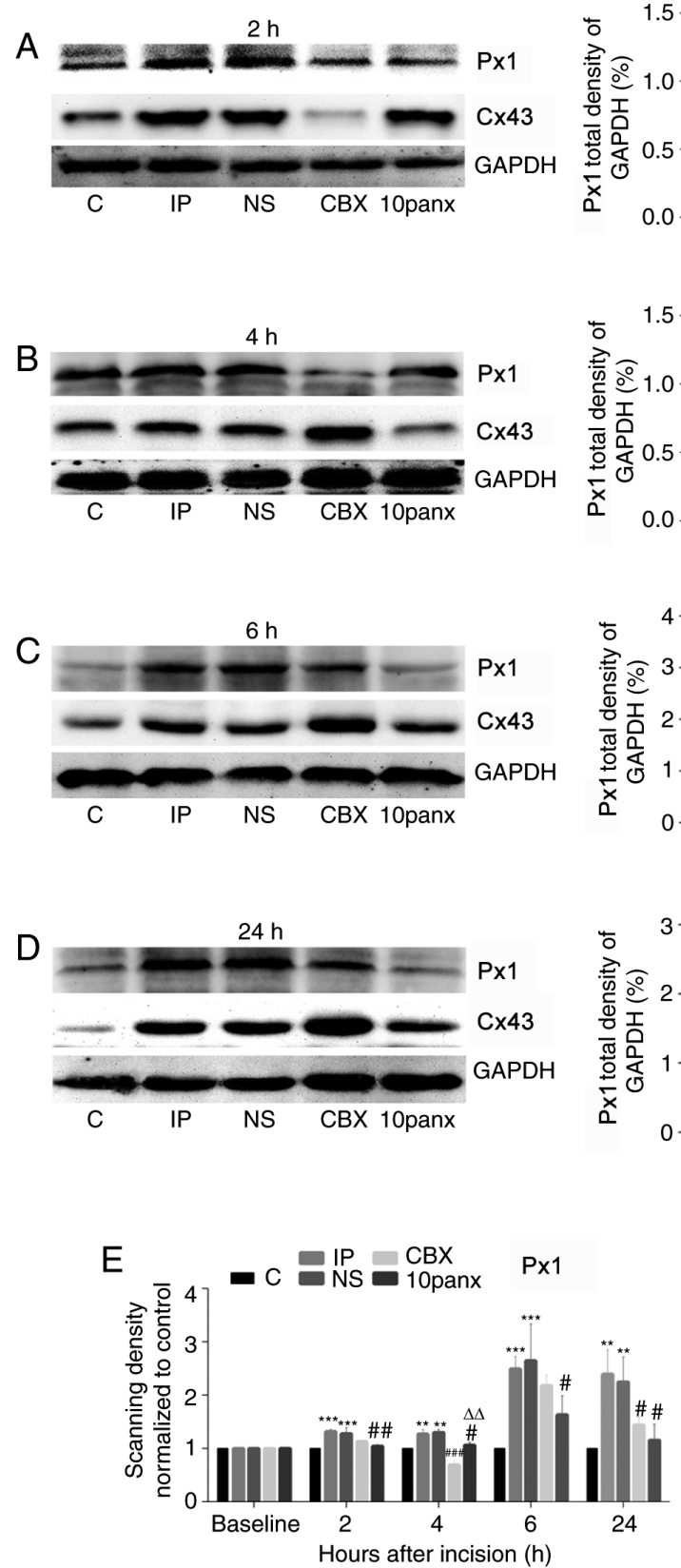
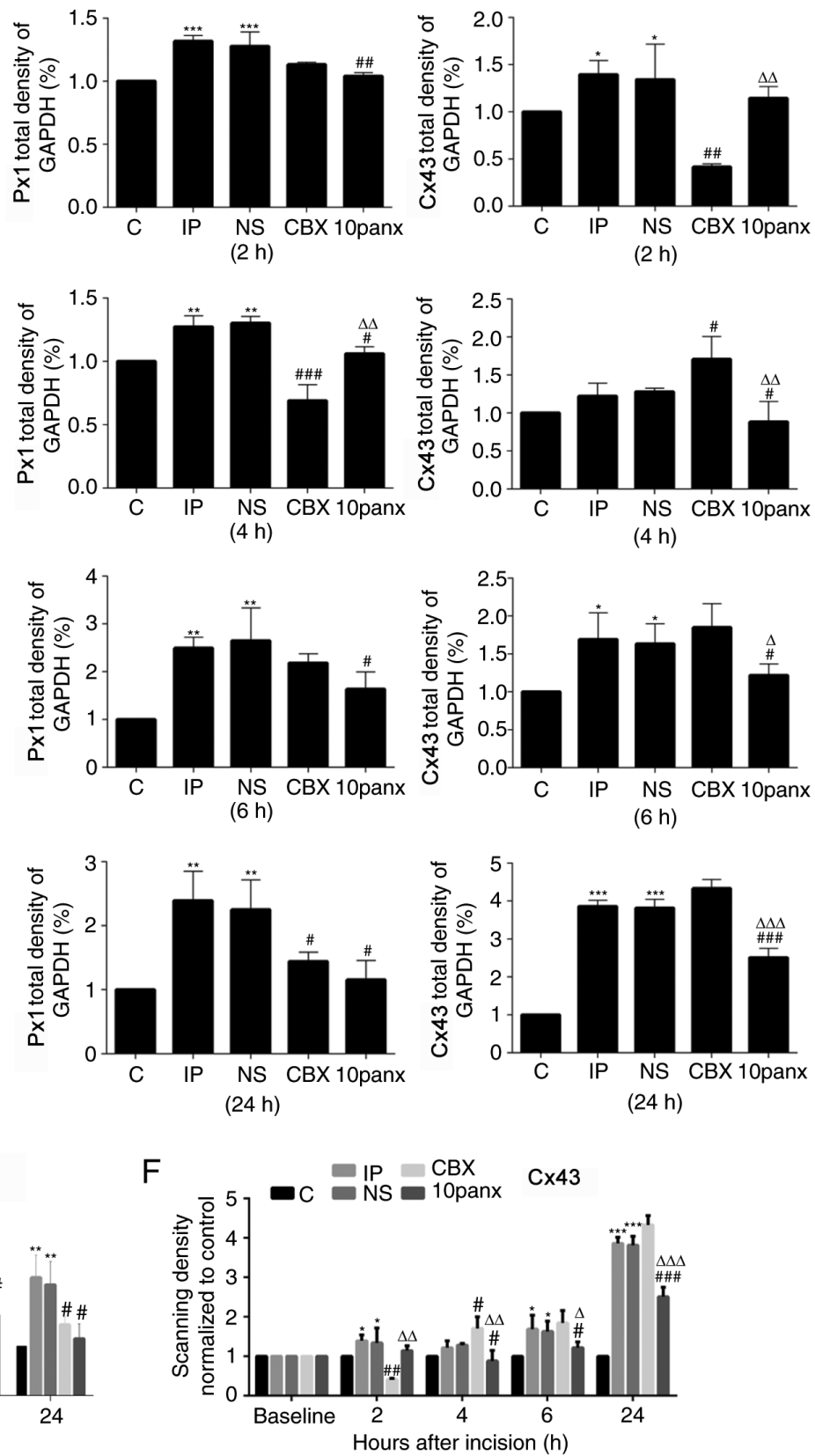

Figure 2. Px1 and Cx43 expression in the spinal cords of IP model rats. Expression of Px1 and Cx42 in rats was detected via western blotting at (A) 2, (B) 4 , (C) 6 and (D) $24 \mathrm{~h}$ post-surgery. The scanning density of (E) Px1 and (F) Cx43 expression normalized to control. ${ }^{*} \mathrm{P}<0.05,{ }^{* * *} \mathrm{P}<0.01$ and ${ }^{* * *} \mathrm{P}<0.001$ vs. C group; ${ }^{\#} \mathrm{P}<0.05,{ }^{\# \#} \mathrm{P}<0.01$ and ${ }^{\# \# "} \mathrm{P}<0.001$ vs. NS group; ${ }^{\circ} \mathrm{P}<0.05,{ }^{\Delta \Delta} \mathrm{P}<0.01$ and ${ }^{\Delta \Delta \Delta} \mathrm{P}<0.001$ vs. CBX group. Data are presented as the mean $\pm \mathrm{SD}$. C, control group; IP, incision pain; NS, normal saline; CBX, carbenoxolone; 10panx, pannexin-1 mimetic inhibitory peptide; Px1, pannexin 1; Cx43, connexin 43.

with adverse side effects (22). The present study showed that CBX could relieve acute surgical pain with a higher specificity and reduced adverse effects. Thus, CBX may have potential to be used as an analgesic in the future.

As a GJ inhibitor, CBX has significant effects in pain reduction (23), ameliorating $\mathrm{Px} 1$ channel activity by regulating the first extracellular loop (16). In addition, CBX has been found to have potential effects on potentially suppressing neuropathic antitumor drug-induced pain by blocking astrocyte GJs and inhibiting the increase of GFAP levels in the spinal cord (18). Collectively, the formation of astrocyte GJs could play a vital role in the transition of acute pain to chronic pain (24-26). In the present study, it was found that inducing an IP model of pain led to an increase in Px1 levels 2, 4, 6 and $24 \mathrm{~h}$ after incision, as well as an increase in Cx43 levels at 2, 6 and $24 \mathrm{~h}$. There are close correlations between the increased expression of PX channels and mechanical pain sensitization (10). In the present study, Px1 and Cx43 expression levels increased in the intumescentia lumbalis of rats post-surgery. Additionally, the IF assay results revealed that Px1 and Cx43 expression was increased in astrocytes in the intumescentia lumbalis of IP rats, indicating that their expression in astrocytes was tightly associated with the production of acute pain. CBX and 10panx pretreatment led to an increase in the pain threshold to a similar degree at both 2 and 4 h post-surgery, suggesting that CBX relieved acute pain possibly by inhibiting Px1 expression. At 6 or $24 \mathrm{~h}$ post-surgery, the expression levels of Px1 remained high compared with 2 h or 4 h post-surgery. Simultaneously, 


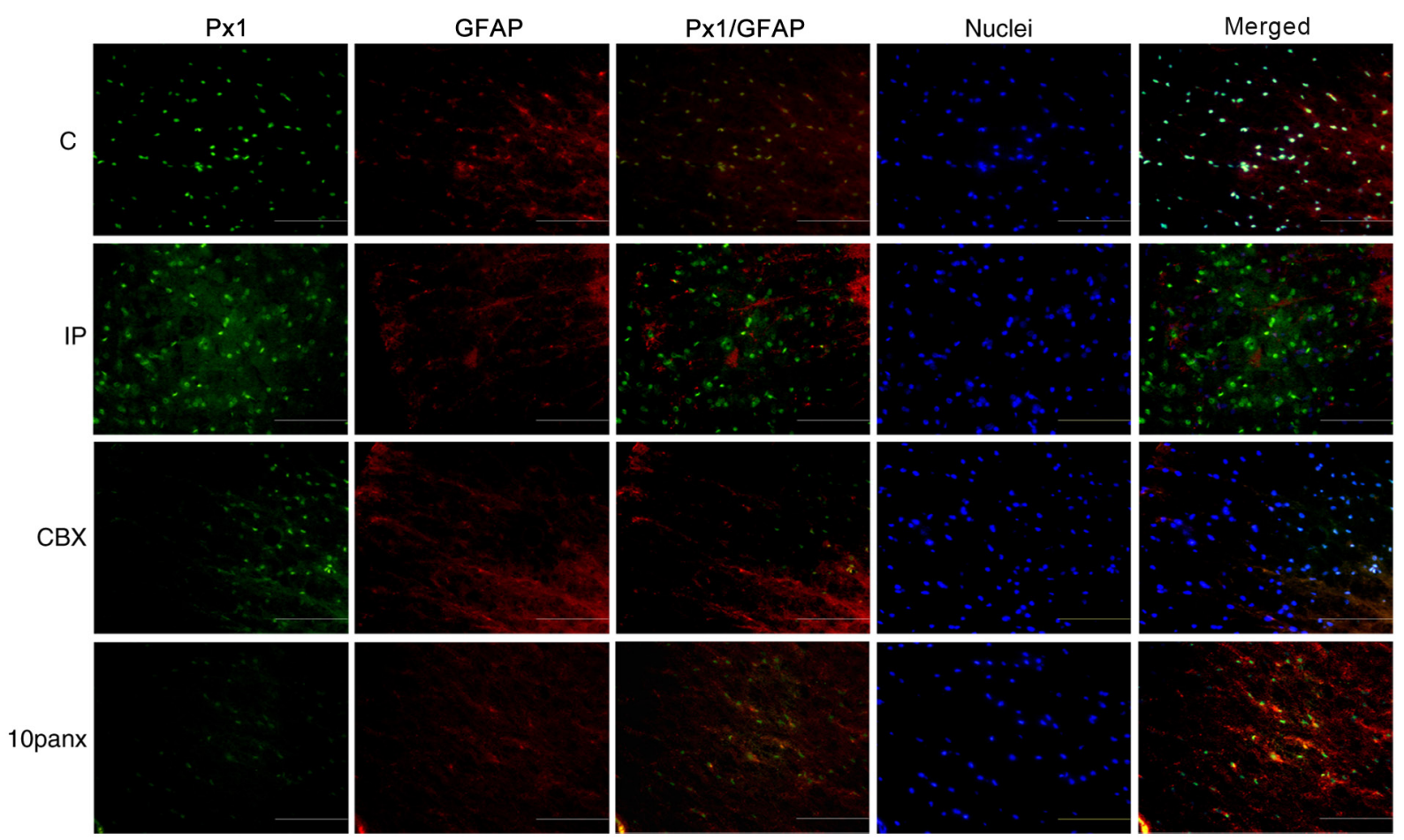

Figure 3. Co-localization of Px1 and GFAP in IP model rats. Px1 (green) and GFAP (red) expression was analyzed using an immunofluorescence assay. Magnification, x200; scale bar, $10 \mu \mathrm{m}$. C, control group; IP, incision pain; CBX, carbenoxolone; 10panx, pannexin-1 mimetic inhibitory peptide; Px1, pannexin 1; GFAP, glial fibrillary acidic protein.

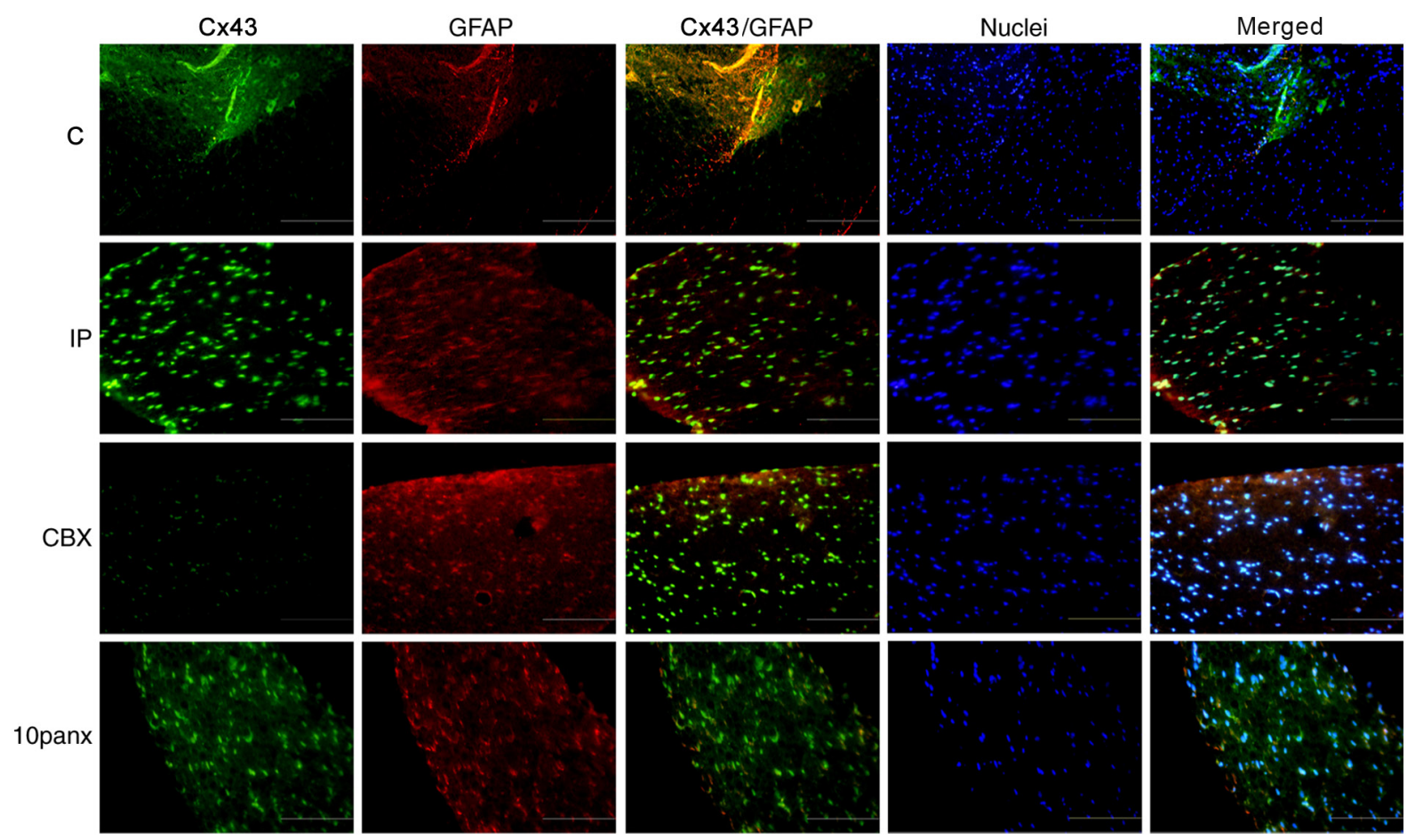

Figure 4. Co-localization of $\mathrm{Cx} 43$ and GFAP in IP model rats. Immunofluorescence assay was used to evaluate the co-localization of Cx43 (green) and GFAP (red). Magnification, x200; scale bar, $10 \mu \mathrm{m}$. C, control group; IP, incision pain; CBX, carbenoxolone; 10panx, pannexin-1 mimetic inhibitory peptide; Cx43, connexin 43; GFAP, glial fibrillary acidic protein.

Cx43 was significantly increased $24 \mathrm{~h}$ after incision compared with the control group. However, the pain threshold value had returned to within normal levels, implying that Px1 and Cx43 play essential roles in the transition of acute to chronic pain.
A previous review indicated that astrocytes both cause and maintain chronic pain (27). GJ regulation, inflammatory factor releases and the activation of specific receptors in astrocytes have been reported to be involved in the development 
A

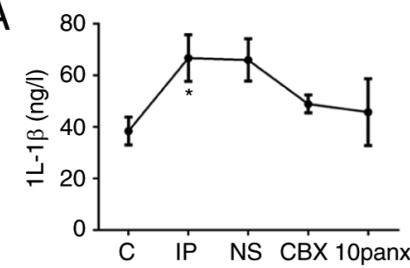

$(2 \mathrm{~h})$

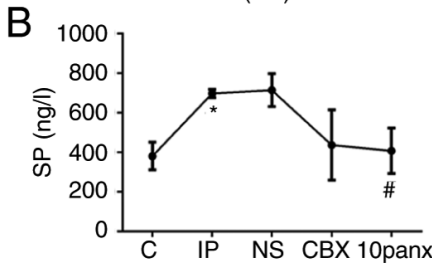

$(2 \mathrm{~h})$

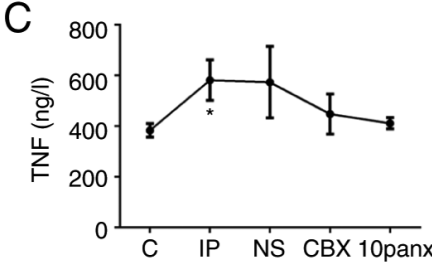

(2 h)
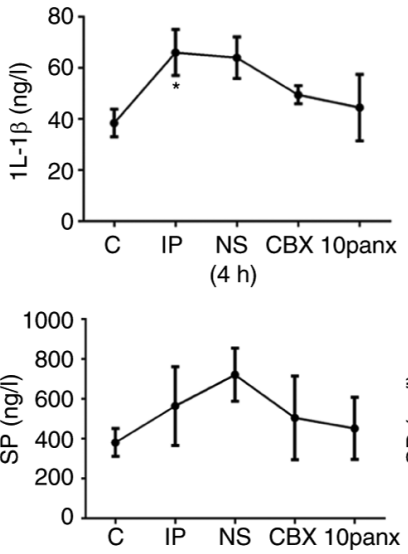

$(4 \mathrm{~h})$

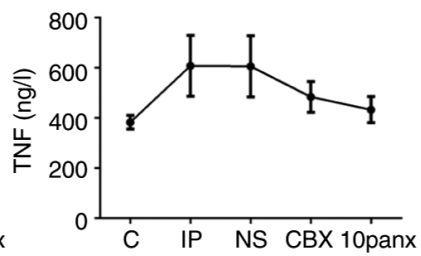

$(4 \mathrm{~h})$
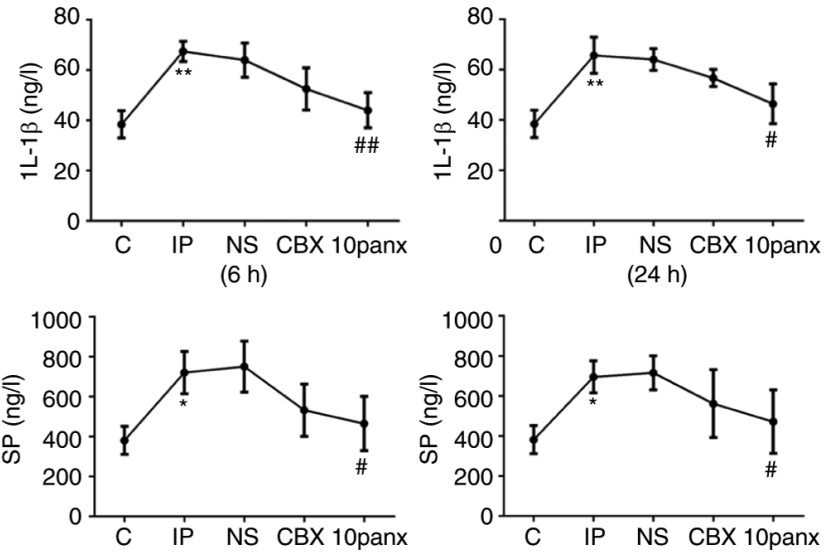

$(6 \mathrm{~h})$

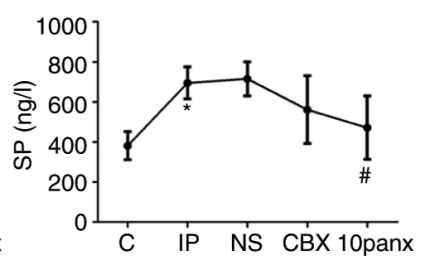

$(24 \mathrm{~h})$

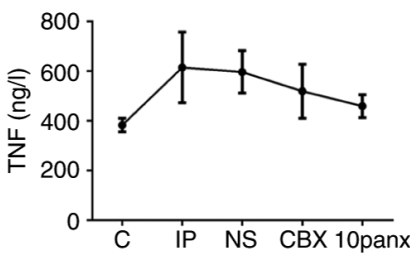

$(6 \mathrm{~h})$

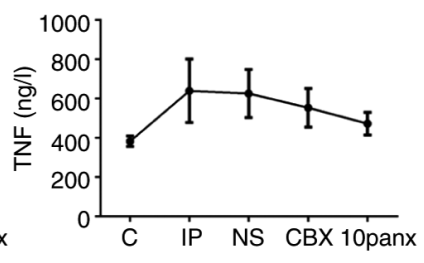

(24 h)

Figure 5. Expression levels of inflammatory markers in IP model rats. Expression levels of (A) IL-1 $\beta$, (B) SP and (C) TNF- $\alpha$ were detected using ELISA kits. ${ }^{*} \mathrm{P}<0.05$ and ${ }^{* *} \mathrm{P}<0.01$ vs. $\mathrm{C}$ group; ${ }^{\#} \mathrm{P}<0.05$ and ${ }^{\# \#} \mathrm{P}<0.01$ vs. IP group. $\mathrm{C}$, control group; IP, incision pain; NS, normal saline; CBX, carbenoxolone; 10 panx, pannexin-1 mimetic inhibitory peptide; SP, P substance.

of pain (28). Px1 and Cx43 have been demonstrated to regulate the release of ATP and glutamate (29). Specifically, CBX-induced ATP release is probably due to the involvement of Panx1 channels in umbilical vein endothelial cells. Cx43 is not involved in the process of ATP release (30). It was found in the present study that compared with the control group, IL-1 $\beta$, SP and TNF- $\alpha$ exhibited relatively high expression levels in the intumescentia lumbalis of IP rats at 2, 4, 6 and $24 \mathrm{~h}$ post-surgery.

Cx43 is specifically expressed in the astrocytes of mammals $(26,31)$. In the present study, the protein expression levels of $\mathrm{Cx} 43$ in the CBX treatment group were significantly lower than those in the IP group at $2 \mathrm{~h}$ post-surgery. Compared with inhibitors, CBX has high specificity, relatively few side effects, and it is easy to manufacture at a low price, thus it has the potential to become a new generation of targeted analgesics (32). 10panx is a mimic peptide of pannexin 1, which suppresses the formation of Px1 channels (33). A previous study suggested that 10panx inhibits neuronal death and the inflammatory response (34), and astrocytic Cx43 is reportedly involved in the development of neuropathic and chronic pain (35-38).

Astrocytes also play important roles in inducing pain in acute pain models. A previous study indicated that astrocytes could cause pain via the release of TNF- $\alpha$ and stromal cell-derived factor 1 (39). Furthermore, IL-1 $\beta$ serves important roles in the production of acute pain, contributing to increased calcium and glutamatergic activities (40). Blocking astrocyte activation has been reported to suppress and ameliorate pain sensitivity (41). Collectively, these findings indicate that $\mathrm{CBX}$ decreases acute pain by regulating Px1 and Cx43 hemichannels, as well as the inflammatory response, which the present study also demonstrated. The mechanism of action of CBX in astrocytes in acute pain, as well as the efficacy of CBX on acute pain caused by other factors, still requires further exploration. In the present study, the drug was administrated through intrathecal administration. Intrathecal administration is an important means of investigating drug mechanisms at the spinal cord level, which increases local drug concentrations (42). In addition, compared with an intrathecal catheter, intrathecal injection is not only simple and convenient with a high success rate, but also rarely results in spinal cord injury or secondary infection (42). In addition, poorly managed acute pain has the potential to progress to chronic pain (43). Taken together, Px1 and Cx43 in astrocytes could be implicated in pain behaviors improved by $\mathrm{CBX}$ and $\mathrm{CBX}$ has the potential to reduce acute pain by decreasing Px1 and Cx43 levels. Px1 and $\mathrm{Cx} 43$ from spinal astrocytes may serve important roles in the early stages and maintenance of acute pain, while preoperative injection of $\mathrm{CBX}$ has the potential to relieve hyperalgesia.

\section{Acknowledgements}

Not applicable.

\section{Funding}

The present study was supported 2016 Master Supervisor Scientific Research Fund of The Second Hospital of Lanzhou University entitled 'Effect of propentofylline on MAPKs signal transduction pathway expression in rats with acute incision pain' (grant no. sdkyjj-15). 


\section{Availability of data and materials}

The datasets used and/or analyzed during the current study are available from the corresponding author on reasonable request.

\section{Authors' contributions}

SD and YS made substantial contributions to conception and design, acquisition of data, analysis interpretation of data, acquisition of funding, collection of data and general supervision of the research group. SD, KZ and YS made substantial contributions to acquisition of data and analysis interpretation of data. SD and KZ confirmed the authenticity of all the raw data. All authors reviewed and approved the final manuscript.

\section{Ethics approval and consent to participate}

All experiments were performed in accordance with the guidance of The International Association for the Study of Pain, and approved by the Ethics Committee of The Second Hospital of Lanzhou University (approval no. D2019-064; Lanzhou, China).

\section{Patient consent for publication}

Not applicable.

\section{Competing interests}

The authors declare that they have no competing interests.

\section{References}

1. Kehlet H: Postoperative pain, analgesia, and recovery-bedfellows that cannot be ignored. Pain 159 (Suppl 1): S11-S16, 2018.

2. Ji RR, Chamessian A and Zhang YQ: Pain regulation by non-neuronal cells and inflammation. Science 354: 572-577, 2016.

3. Peng J, Gu N, Zhou L, B Eyo U, Murugan M, Gan WB and Wu LJ: Microglia and monocytes synergistically promote the transition from acute to chronic pain after nerve injury. Nat Commun 7 : 12029, 2016.

4. Ramani M, Mylvaganam S, Krawczyk M, Wang L, Zoidl C, Brien J, Reynolds JN, Kapur B, Poulter MO, Zoidl G and Carlen PL: Differential expression of astrocytic connexins in a mouse model of prenatal alcohol exposure. Neurobiol Dis 91: 83-93, 2016.

5. Dahl G and Muller KJ: Innexin and pannexin channels and their signaling. FEBS Lett 588: 1396-1402, 2014.

6. Garre JM, Yang G, Bukauskas FF and Bennett MV: FGF-1 triggers pannexin-1 hemichannel opening in spinal astrocytes of rodents and promotes inflammatory responses in acute spinal cord slices. J Neurosci 36: 4785-4801, 2016.

7. Bargiotas P, Krenz A, Hormuzdi SG, Ridder DA, Herb A Barakat W, Penuela S, von Engelhardt J, Monyer $\mathrm{H}$ and Schwaninger M: Pannexins in ischemia-induced neurodegeneration. Proc Natl Acad Sci USA 108: 20772-20777, 2011.

8. Reyes EP, Cerpa V, Corvalán L and Retamal MA: Cxs and Panx-hemichannels in peripheral and central chemosensing in mammals. Front Cell Neurosci 8: 123, 2014.

9. Zhang Y, Laumet G, Chen SR, Hittelman WN and Pan HL: Pannexin-1 Up-regulation in the dorsal root ganglion contributes to neuropathic pain development. J Biol Chem 290: 14647-14655, 2015.

10. Burma NE, Bonin RP, Leduc-Pessah H, Baimel C, Cairncross ZF, Mousseau M, Shankara JV, Stemkowski PL, Baimoukhametova D, Bains JS, et al: Blocking microglial pannexin-1 channels alleviates morphine withdrawal in rodents. Nat Med 23: 355-360, 2017.
11. Hanstein R, Hanani M, Scemes E and Spray DC: Glial pannexin1 contributes to tactile hypersensitivity in a mouse model of orofacial pain. Sci Rep 6: 38266, 2016.

12. Bravo D, Ibarra P, Retamal J, Pelissier T, Laurido C, Hernandez A and Constandil L: Pannexin 1: A novel participant in neuropathic pain signaling in the rat spinal cord. Pain 155: 2108-2115, 2014.

13. Michalski $\mathrm{K}$ and Kawate T: Carbenoxolone inhibits Pannexin1 channels through interactions in the first extracellular loop. J Gen Physiol 147: 165-174, 2016.

14. Morioka N, Nakamura Y, Zhang FF, Hisaoka-Nakashima K and Nakata Y: Role of connexins in chronic pain and their potential as therapeutic targets for next-generation analgesics. Biol Pharm Bull 42: 857-866, 2019.

15. D'Hondt C, Iyyathurai J, Himpens B, Leybaert L and Bultynck G: Cx43-hemichannel function and regulation in physiology and pathophysiology: Insights from the bovine corneal endothelial cell system and beyond. Front Physiol 5: 348, 2014.

16. Dierks A, Bader A, Lehrich T and Ngezahayo A: Stimulation of the $A_{2 B}$ adenosine receptor subtype enhances connexin 26 hemichannel activity in small airway epithelial cells. Cell Physiol Biochem 53: 606-622, 2019.

17. Treede RD: The international association for the study of pain definition of pain: As valid in 2018 as in 1979, but in need of regularly updated footnotes. Pain Rep 3: e643, 2018.

18. Roh DH, Yoon SY, Seo HS, Kang SY, Han HJ, Beitz AJ and Lee JH: Intrathecal injection of carbenoxolone, a gap junction decoupler, attenuates the induction of below-level neuropathic pain after spinal cord injury in rats. Exp Neurol 224: 123-132, 2010.

19. Brennan TJ, Vandermeulen EP and Gebhart GF: Characterization of a rat model of incisional pain. Pain 64: 493-502, 1996.

20. Zhao H, Alam A, Chen Q, A Eusman M, Pal A, Eguchi S, $\mathrm{Wu} \mathrm{L}$ and $\mathrm{Ma} \mathrm{D}$ : The role of microglia in the pathobiology of neuropathic pain development: What do we know? Br J Anaesth 118: 504-516, 2017.

21. Chu LW, Cheng KI, Chen JY, Cheng YC, Chang YC, Yeh JL, Hsu JH, Dai ZK and Wu BN: Loganin prevents chronic constriction injury-provoked neuropathic pain by reducing TNF- $\alpha /$ IL- $1 \beta$-mediated NF- $\kappa$ B activation and Schwann cell demyelination. Phytomedicine 67: 153166, 2020.

22. de Boer HD, Detriche $\mathrm{O}$ and Forget P: Opioid-related side effects: Postoperative ileus, urinary retention, nausea and vomiting, and shivering. A review of the literature. Best Pract Res Clin Anaesthesiol 31: 499-504, 2017.

23. Deng GC, Lu M, Zhao YY, Yuan Y and Chen G: Activated spinal astrocytes contribute to the later phase of carrageenan-induced prostatitis pain. J Neuroinflammation 16: 189, 2019.

24. Robinson CR and Dougherty PM: Spinal astrocyte gap junction and glutamate transporter expression contributes to a rat model of bortezomib-induced peripheral neuropathy. Neuroscience 285: 1-10, 2015.

25. Wieseler-Frank J, Maier SF and Watkins LR: Glial activation and pathological pain. Neurochem Int 45: 389-395, 2004.

26. Chen MJ, Kress B, Han X, Moll K, Peng W, Ji RR and Nedergaard M: Astrocytic CX43 hemichannels and gap junctions play a crucial role in development of chronic neuropathic pain following spinal cord injury. Glia 60: 1660-1670, 2012.

27. O'Callaghan JP and Miller DB: Spinal glia and chronic pain. Metabolism 59 (Suppl 1): S21-S26, 2010.

28. Xing L, Yang T, Cui S and Chen G: Connexin hemichannels in astrocytes: Role in CNS disorders. Front Mol Neurosci 12: 23, 2019.

29. Wei H, Deng F, Chen Y, Qin Y, Hao Y and Guo X: Ultrafine carbon black induces glutamate and ATP release by activating connexin and pannexin hemichannels in cultured astrocytes. Toxicology 323: 32-41, 2014.

30. Gdecke S, Roderigo C, Rose CR, Rauch BH, Gödecke A and Schrader J: Thrombin-induced ATP release from human umbilical vein endothelial cells. Am J Physiol Cell Physiol 302: C915-C923, 2012.

31. Yoon SY, Robinson CR, Zhang H and Dougherty PM: Spinal astrocyte gap junctions contribute to oxaliplatin-induced mechanical hypersensitivity. J Pain 14: 205-214, 2013.

32. Di Cesare Mannelli L, Marcoli M, Micheli L, Zanardelli M, Maura G, Ghelardini C and Cervetto C: Oxaliplatin evokes P2X7-dependent glutamate release in the cerebral cortex: A pain mechanism mediated by Pannexin 1. Neuropharmacology 97: 133-141, 2015. 
33. Basova LV, Tang X, Umasume T, Gromova A, Zyrianova T, Shmushkovich T, Wolfson A, Hawley D, Zoukhri D, Shestopalov VI and Makarenkova HP: Manipulation of panx1 activity increases the engraftment of transplanted lacrimal gland epithelial progenitor cells. Invest Ophthalmol Vis Sci 58: 5654-5665, 2017.

34. Wei R, Bao W, He F, Meng F, Liang H and Luo B: Pannexin1 channel inhibitor $\left({ }^{10}\right.$ panx $)$ protects against transient focal cerebral ischemic injury by inhibiting RIP3 expression and inflammatory response in rats. Neuroscience 437: 23-33, 2020.

35. Wang $\mathrm{A}$ and $\mathrm{Xu} \mathrm{C}$ : The role of connexin 43 in neuropathic pain induced by spinal cord injury. Acta Biochim Biophys Sin (Shanghai) 51: 555-561, 2019

36. Zhou L, Ao L, Yan Y, Li C, Li W, Ye A, Liu J, Hu Y, Fang W and $\mathrm{Li} \mathrm{Y}$ : Levo-corydalmine attenuates vincristine-induced neuropathic pain in mice by upregulating the $\mathrm{Nrf} 2 / \mathrm{HO}-1 / \mathrm{CO}$ pathway to inhibit connexin 43 expression. Neurotherapeutics 17: 340-355, 2020.

37. Vicario N, Pasquinucci L, Spitale FM, Chiechio S, Turnaturi R, Caraci F, Tibullo D, Avola R, Gulino R, Parenti R and Parenti C: Simultaneous activation of $\mathrm{Mu}$ and Delta opioid receptors reduces allodynia and astrocytic connexin 43 in an animal model of neuropathic pain. Mol Neurobiol 56: 7338-7354, 2019.

38. Jin YZ, Zhang P, Hao T, Wang LM, Guo MD and Gan YH: Connexin 43 contributes to temporomandibular joint inflammation induced-hypernociception via sodium channel 1.7 in trigeminal ganglion. Neurosci Lett 707: 134301, 2019.
39. Liu X, Tonello R, Ling Y, Gao YJ and Berta T: Paclitaxel-activated astrocytes produce mechanical allodynia in mice by releasing tumor necrosis factor- $\alpha$ and stromal-derived cell factor 1 . J Neuroinflammation 16: 209, 2019.

40. Yan X, Li F, Maixner DW, Yadav R, Gao M, Ali MW, Hooks SB and Weng HR: Interleukin-1beta released by microglia initiates the enhanced glutamatergic activity in the spinal dorsal horn during paclitaxel-associated acute pain syndrome. Glia 67: 482-497, 2019.

41. Obata H, Sakurazawa S, Kimura M and Saito S: Activation of astrocytes in the spinal cord contributes to the development of bilateral allodynia after peripheral nerve injury in rats. Brain Res 1363: 72-80, 2010.

42. Li X, Sun S, Wang Q and Zhao Z: Population pharmacokinetics of combined intravenous and local intrathecal administration of meropenem in aneurysm patients with suspected intracranial infections after craniotomy. Eur J Drug Metab Pharmacokinet 43: 45-53, 2018

43. Ossipov MH, Morimura K and Porreca F: Descending pain modulation and chronification of pain. Curr Opin Support Palliat Care 8: 143-151, 2014.

c) (i) $\odot$ This work is licensed under a Creative Commons Attribution-NonCommercial-NoDerivatives 4.0 International (CC BY-NC-ND 4.0) License. 